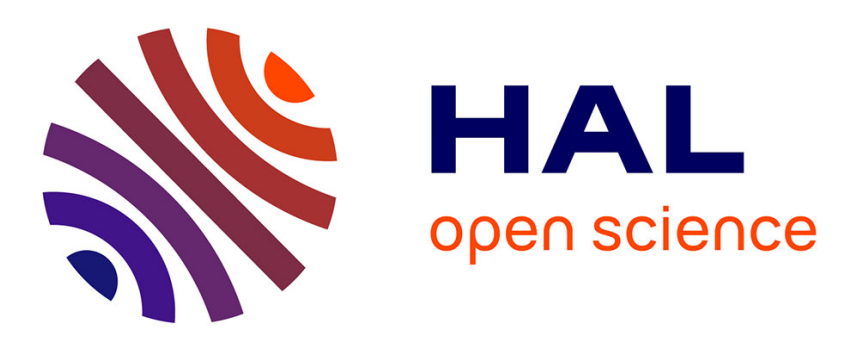

\title{
A UAS site substitution approach to the in vivo dissection of promoters: interplay between the GATAb activator and the AEF-1 repressor at a Drosophila ecdysone response unit
}

Véronique Brodu, Bruno Mugat, Pierre Fichelson, Jean-Antoine Lepesant, Christophe Antoniewsk

\section{To cite this version:}

Véronique Brodu, Bruno Mugat, Pierre Fichelson, Jean-Antoine Lepesant, Christophe Antoniewsk. A UAS site substitution approach to the in vivo dissection of promoters: interplay between the GATAb activator and the AEF-1 repressor at a Drosophila ecdysone response unit. Development (Cambridge, England), 2001. hal-03030908

\section{HAL Id: hal-03030908 https://hal.science/hal-03030908}

Submitted on 30 Nov 2020

HAL is a multi-disciplinary open access archive for the deposit and dissemination of scientific research documents, whether they are published or not. The documents may come from teaching and research institutions in France or abroad, or from public or private research centers.
L'archive ouverte pluridisciplinaire HAL, est destinée au dépôt et à la diffusion de documents scientifiques de niveau recherche, publiés ou non, émanant des établissements d'enseignement et de recherche français ou étrangers, des laboratoires publics ou privés. 


\title{
A UAS site substitution approach to the in vivo dissection of promoters: interplay between the GATAb activator and the AEF-1 repressor at a Drosophila ecdysone response unit
}

\author{
Véronique Brodu ${ }^{1, *}$, Bruno Mugat ${ }^{2}$, Pierre Fichelson ${ }^{3}$, Jean-Antoine Lepesant ${ }^{1}$ \\ and Christophe Antoniewski ${ }^{1, \neq}$ \\ ${ }^{1}$ Institut Jacques-Monod, CNRS UMR7592, Université Paris 6 P. et M. Curie, Université Paris 7-Denis-Diderot, 2, place Jussieu, \\ F-75251, Paris cedex 05, France \\ 2Institut de Génétique Humaine, CNRS, 141, rue de la Cardonille, F-34396 Montpellier, cedex 5, France \\ ${ }^{3}$ Laboratoire de Biologie du Développement, Université Paris 6-P et M. Curie, 9 quai St Bernard, case 24, F-75005 Paris, France \\ *Present address: Medical Research Council, National Institute for Medical Research, The Ridgeway, Mill Hill, London, NW7 1AA, UK \\ ¥Author for correspondence (e-mail: antoniewski@ijm.jussieu.fr) \\ Accepted 17 April 2001
}

\section{SUMMARY}

An ecdysone response unit (EcRU) directs the expression of the Fat body protein 1 (Fbp1) gene in the third instar larval Drosophila fat body. The tissue-specific activity of this regulatory element necessitates the binding of both the ligand-activated EcR/USP ecdysone receptor and GATAb. To analyze the role played by GATAb in the regulation of the Fbp1 EcRU activity, we have replaced the GATAbinding sites GBS1, GBS2 and GBS3 in the Fbp1 EcRU with UAS sites for the yeast GAL4 activator and tested the activity of the mutagenized Fbp1 EcRUs in transgenic lines, either in the presence or absence of ubiquitously expressed GAL4. Our results reveal that GATAb plays two distinguishable roles at the Fbp1 EcRU that contribute to the tissue-specific activity of this regulatory element. On the one hand, GATAb mediates a fat body-specific transcriptional activation. On the other hand, it antagonizes specifically in the fat body a ubiquitous repressor that maintains the Fbp1 EcRU in an inactive state, refractory to activation by GAL4. We identified this repressor as AEF-1, a factor previously shown to be involved in the regulation of the Drosophila Adh and yp1-yp2 genes. These results show that, for a functional dissection of complex promoter-dependent regulatory pathways, the replacement of specific regulatory target sites by UAS GAL4 binding sites is a powerful alternative to the widely used disruption approach.

Key words: Drosophila, Ecdysone, Nuclear receptor, GATAb, serpent, AEF-1, Fbpl

\section{INTRODUCTION}

The specific gene transcription patterns formed during development of metazoans result from the activity of the cisregulatory modules that control gene promoters. Even when these cis-regulatory modules are responsible for elementary transcription patterns, as for example in a single tissue during a short period of development, they are always composed of multiple target sites for transcription factors (Arnone and Davidson, 1997; Kirchhamer et al., 1996). How the activities of transcription factors are integrated at the level of cisregulatory modules to produce highly specific regulatory outputs remains a central issue in developmental biology.

Hormone response units (HRU) in nuclear receptorregulated promoters constitute a particular class of cisregulatory modules. They are composed of an assembly of binding sites for a variety of transcription factors, including hormone receptor-binding sites, and determine in which tissue(s) and during which developmental period(s) a gene will respond to the hormone (Lucas and Granner, 1992). Drosophila melanogaster is a choice animal for the study of HRUs in the context of a developing organism. At the end of the third larval instar, a pulse of the steroid hormone 20hydroxyecdysone (hereafter referred to as ecdysone) activates the ecdysone receptor, which is composed of a heterodimer between the nuclear receptors EcR and USP (Koelle et al., 1991; Thomas et al., 1993; Yao et al., 1993). This receptor in turn differentially regulates a number of primary ecdysone response genes in different target tissues (Andres and Thummel, 1992). This hormonally controlled genetic program triggers puparium formation and initiates metamorphosis.

We are interested in understanding the molecular mechanisms whereby ecdysone response units (EcRUs) integrate multiple regulatory inputs to mediate distinct tissue- and time-specific transcriptional responses to circulating ecdysone. Our model gene Fat body protein 1 ( Fbpl), which encodes a receptor mediating the uptake of hexamerins from the hemolymph by the larval fat body (Burmester et al., 1999), is transcribed 
exclusively in this tissue in response to the late third instar ecdysone peak (Andres et al., 1993; Lepesant et al., 1986). Germline transformation analysis has allowed the delimitation in the proximal Fbpl promoter of an EcRU that is composed of two separable regions (Fig. 1). A 70 bp enhancer (E) is sufficient in itself to specify the spatially and temporally correct ecdysonecontrolled pattern of $F b p l$ expression (Laval et al., 1993) and an upstream region (A) contains a $32 \mathrm{bp}$ sequence shown to amplify at least fivefold the specific transcriptional response conferred by the enhancer (Lapie et al., 1993).

The EcR/USP ecdysone receptor binds to a pseudopalindromic site (EBS) in the central part of the $F b p l$ enhancer (Antoniewski et al., 1994). This site is strictly necessary, but not sufficient by itself, for the activation of a lacZ reporter transgene by the Fbpl EcRU in the late third instar fat body (Antoniewski et al., 1996). Recently, we replaced EBS with a UAS site for the yeast activator GAL4. Ubiquitously expressed GAL4 precociously activated the modified EcRU throughout the third larval instar, but, strikingly, the expression of the lacZ reporter transgene remained fat body specific (Brodu et al., 1999). Hence, this UAS substitution approach has revealed that factors other than the EcR/USP ecdysone receptor are targeted to the EcRU and able to restrict spatially its activity to the fat body, even when the receptor is replaced by the ubiquitously expressed and autonomous GAL4 transcriptional activator.

Our previous work had suggested that this factor was perhaps GATAb, a member of the GATA family encoded by the serpent (srp) gene (Reuter, 1994b). GATAb is required for the embryonic development of the endodermal gut and hematopoietic tissues and is involved in the control of the differentiation of the fat body (Lebestky et al., 2000; Moore et al., 1998; Rehorn et al., 1996; Riechmann et al., 1998; Sam et al., 1996). We have shown that GATAb is expressed in a subset of larval tissues, including the fat body, during the third instar, and that it binds in vitro to three sites, GBS1, GBS2 and GBS3, flanking EBS in the Fbpl enhancer (Brodu et al., 1999). Mutagenesis of these sites, as well as overexpression of GATAb, demonstrate that binding of GATAb to GBS1 is strictly necessary for the activity of the Fbpl EcRU and contributes to its tissue specificity (Brodu et al., 1999).

We further applied the UAS substitution approach to a detailed investigation of the contribution of GATAb to the highly tissue-specific activity of the Fbpl EcRU. Unexpectedly, we found that ubiquitously expressed GAL4 could replace GATAb at GBS1 without any change in the tissue specificity of the FbpI EcRU. Further combinations of GBS substitutions by UAS enabled us to show that this was due to a redundant tissuespecific role of GATAb through its binding to GBS3 and revealed that GATAb fulfilled two distinct functions at the Fbpl EcRU: mediating a fat body-specific transcriptional activation and antagonizing (specifically in the fat body) the ubiquitous AEF1 repressor that maintains the $F b p l$ EcRU in an inactive state in other tissues. This points to a mode of repression exerted by AEF-1 that has not been described previously.

\section{MATERIALS AND METHODS}

\section{Plasmids}

The germline transformation vector pAE was as described previously (Antoniewski et al., 1996) where it was referred to as pAEP1. All the mutated constructs were derived from pAE by PCR mutagenesis and named using the following nomenclature. UAS1 designates the disruption of GBS1 by replacement of the ${ }_{-130} \mathrm{CTGA}_{-127}$ sequence by a CGGAGTACTGTCCTCCG UAS site. UAS2-3 designates the disruption of both GBS2 and GBS3 by replacement of the -89TCGGGAGTCAAGCGATAGGCC_-69 sequence by a unique CGCGGAGTACTGTCCTCCG UAS site. UAS ${ }^{\mathrm{EBS}}$ designates the disruption of the ecdysone receptor binding site by replacement of the -104ATTCATTCAAC_-94 sequence by the CGGAGTACTGTCCTCCG UAS site. GBS1 ${ }^{\mathrm{m}}, \mathrm{GBS}^{\mathrm{m}}$ and $\mathrm{GBS}^{\mathrm{m}}$ designate 4 bp mutations in GBS1, GBS2 and GBS3, respectively, that have been described previously and shown to abolish the in vitro binding of GATAb (Brodu et al., 1999). EBS $^{m}$ designates a 4 bp mutation of EBS shown to abolish the binding of EcR/USP (mutation $\delta$ in Antoniewski et al., 1994). AEFm designates a 7 bp mutation of the AEF-1 site in the Fbpl EcRU (as indicated in Fig. 5). In the pE[UAS1-UAS2-3] construct, GBS1, GBS2 and GBS3 were replaced by UAS sites as previously indicated, and the Fbpl EcRU was deleted from its element A (sequence between -194 and -139). The p5UAS-Fbpl-lacZ was constructed by inserting in the unique $B g l$ II site of $\mathrm{pBP} 1$, a doublestranded oligonucleotide containing five tandemly repeated UAS sites separated by the dinucleotide AG and flanked by BamHI and $B g l \mathrm{II}$ sites.

\section{Germline transformations and GAL4 substitution experiments}

Transgenic lines for the various constructs were established as described by Brodu et al. (Brodu et al., 1999). At least three independent lines for each construct were tested and analyzed. The GAL4-UAS system (Brand and Perrimon, 1993) was used to study the effect of GAL4-to-GATAb and GAL4-to-EcR/USP substitutions. This was tested by crossing lines homozygous for the AE derivative constructs with the homozygous GAL4 ${ }^{\text {daG32 }}$ line that expresses GAL4 under the control of the daughterless promoter (Brodu et al., 1999; Wodarz et al., 1995).

\section{Histochemical assays of $\beta$-galactosidase activity}

Drosophila stocks were maintained at $25^{\circ} \mathrm{C}$ on a standard Drosophila medium. Developmental-stage determination of larvae was carried out as described (Andres and Thummel, 1994) and the histochemical staining assay of $\beta$-galactosidase activity was performed essentially as previously described (Ashburner, 1989) using X-Gal.

\section{Gel shift assays}

DNA-binding reactions and subsequent gel electrophoresis were performed using $4 \mu \mathrm{l}$ of late third instar fat body nuclear extract as described previously (Antoniewski et al., 1994). Sequences of the DNA probe and competitors are depicted in Fig. 5. Rabbit polyclonal antibody $(1 \mu \mathrm{l})$ raised against AEF-1 (kindly provided by T. Maniatis) and $1 \mu \mathrm{g}$ of protein $\mathrm{A}$ (when indicated) were added to the binding reactions for the supershift experiments.

\section{RESULTS}

\section{GAL4 can take the place of GATAb at GBS1 in the tissue-specific activation of the Fbp1 promoter}

We had shown previously that the AE construct in which the Fbpl EcRU (-194 to -69) drives the minimal Fbpl promoter $(-68$ and +80$)$ fused to the lac $Z$ reporter gene was expressed exclusively in the fat body of late third instar transgenic larvae in response to ecdysone (Fig. 1A; Antoniewski et al., 1996). To further test the contribution of GATAb to the tissue-specific activity of the Fbpl EcRU, we replaced the GBS1 site with a UAS site in this construct. As expected from our mutagenesis 
analysis of the Fbpl EcRU, which had revealed the essential role of GBS1 in its in vivo activity (Brodu et al., 1999), this substitution led to a total inactivation of the resulting AE[UAS1] construct (Fig. 2A). However, when the AE[UAS1] transgene was crossed in a GAL4daG32 line in which GAL4 was expressed in all tissues throughout development, lac $Z$ expression was specifically restored in the fat body of late third instar larvae only (Fig. 2B). This result strongly suggested that sequences flanking GBS1 were bound by transcription factors that had the capacity to modulate, in a tissue-specific manner, the transactivation brought about by GAL4 when it was targeted to GBS1.

Expression patterns of two additional constructs led us to search for such sequences within the enhancer element itself between positions -69 and -138 . Firstly, with the E construct, in which the Fbpl EcRU was deleted from the upstream element $\mathrm{A}$, it could be excluded that sequences essential for tissue specification were located in this region. As determined by histochemical staining and quantitative assay of $\beta$ galactosidase activity in four independent lines, the E construct was expressed at a much lower level than the AE construct (Fig. 1B and data not shown) in agreement with a deletion analysis that had shown that element $\mathrm{A}$ amplifies at least fivefold the transcriptional response conferred by the Fbpl enhancer alone (Lapie et al., 1993). However, this expression remained restricted to the late third instar fat body.

Similarly, when the 5UAS-Fbpl-lacZ construct with five UAS sites fused upstream of the $F b p l$ minimal promoter was crossed in the GAL4daG32 line, the lac $Z$ reporter gene was expressed in all tissues except the central nervous system and gut (Fig. 2G). This result excluded the possibility that sequences responsible for a fat body-specific modulation of GAL4 activation were present in the minimal promoter downstream from the enhancer.

\section{Functional redundancy of GBS1 and GBS3}

In order to pursue the application of the UAS substitution approach to a further functional dissection of element $\mathrm{E}$, we first ruled out the possibility that the ecdysone receptor EcR/USP played a direct role in the tissue-specificity of GAL4 transactivation by testing the AE[UAS1-UAS ${ }^{\mathrm{EBS}}$ ] construct in which both the GBS1 and EBS sites were replaced by UAS sites. When crossed in the GAL4 ${ }^{\mathrm{daG} 32}$ line, this construct was still specifically expressed in the fat body of third instar larvae (Fig. 2C), indicating that sequences other than those of GBS1 and EBS in the Fbpl EcRU were responsible for the tissuespecific modulation of GAL4 activity.

In our previous mutagenesis analysis we found that GBS2 and GBS3 were not essential for the activity of the Fbpl EcRU when GBS1 was left intact. We showed, however, that they were bound in vitro by GATAb in fat body nuclear extracts (Brodu et al., 1999). This raised the possibility that the binding of GATAb to these sites was responsible for the restricted
GAL4 transactivation of AE[UAS1] in the late third instar fat body. To test this hypothesis, GBS2, GBS3 or both GBS2 and GBS3 were disrupted in the AE[UAS1] construct and transgenic lines for the resulting constructs AE[UAS1$\mathrm{GBS}^{\mathrm{m}}$ ], AE[UAS1-GBS3 $\left.{ }^{\mathrm{m}}\right]$ and AE[UAS1-GBS2 $\left.{ }^{\mathrm{m}}-\mathrm{GBS}^{\mathrm{m}}{ }^{\mathrm{H}}\right]$ were established. When crossed in the GAL4daG32 line, AE[UAS1-GBS2m] was still expressed specifically in the late third instar fat body (Fig. 2D). In contrast, no expression of AE[UAS1-GBS3 $\left.{ }^{m}\right]$ and AE[UAS1-GBS2m-GBS3 $\left.{ }^{m}\right]$ was observed at any time during development in the same genetic background (Fig. 2E,F). This led us to conclude that, like GBS1, GBS3 possibly plays a role in the tissue-specificity of the Fbpl EcRU activity which could only be revealed when the prevalent GBS1 was inactivated by the UAS substitution. In contrast, GBS2 did not seem able to play the same functional role.

The demonstration of the requirement for GBS3 in the modulation of GAL4 activity when this factor was targeted to a UAS site replacing GBS1, prompted us to analyze the symmetrical situation in which GAL4 was targeted to a UAS site replacing GBS2/GBS3, while GBS1 was either left intact or disrupted. As expected from our previous finding that neither GBS2 nor GBS3 were essential for the activity of the Fbpl EcRU (Brodu et al., 1999), the AE[UAS2-3] transgenic construct, which carried a UAS site in place of both GBS2 and GBS3, was expressed in late third instar fat body, even in the absence of GAL4 (Fig. 3A). When crossed in the GAL4daG32 line, the expression of AE[UAS2-3] was strongly reinforced but remained restricted to the late third instar fat body (Fig. 3B), indicating that this further transactivation of the construct brought about by GAL4 was modulated in a tissue-specific manner. In contrast, the $\mathrm{AE}\left[\mathrm{GBS} 1^{\mathrm{m}}-\mathrm{UAS} 2-3\right]$ construct in which both GBS2 and GBS3 were replaced by a UAS site and GBS1 was disrupted, remained completely silent, even when crossed in the GAL4daG32 line (Fig. 3C). 
Altogether, these results indicate that GAL4 activation of the Fbpl EcRU is dependent upon the integrity of at least one of the two GBS1 or GBS3 sites. This conclusion was confirmed by the AE[UAS1-UAS2-3] construct in which all three
GATAb-binding sites were replaced by two UAS sites. This construct was not expressed at any time during development, even when crossed in the GAL4 $4^{d a G 32}$ line (Fig. 4A; data not shown).
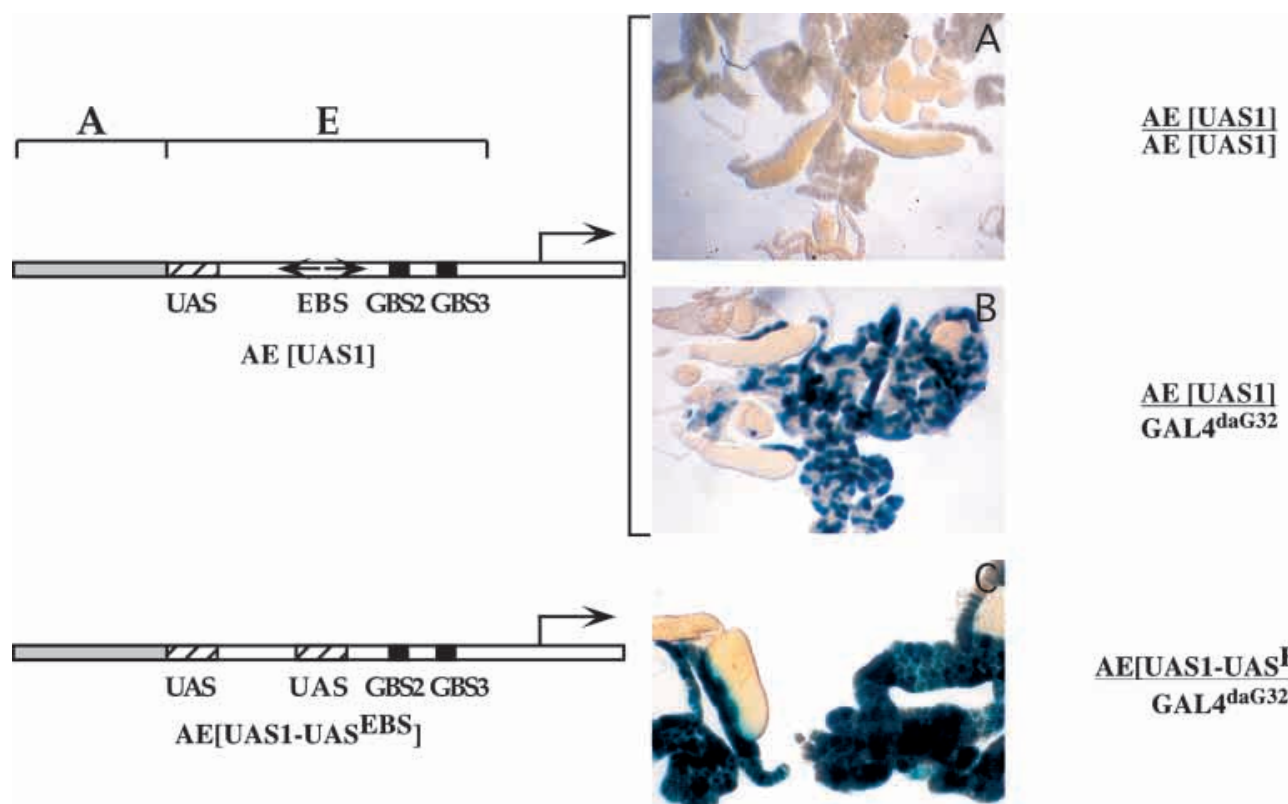

$\frac{\text { GAL4 }^{\text {daG32 }}}{\text { AE[UAS1-UAS }}$
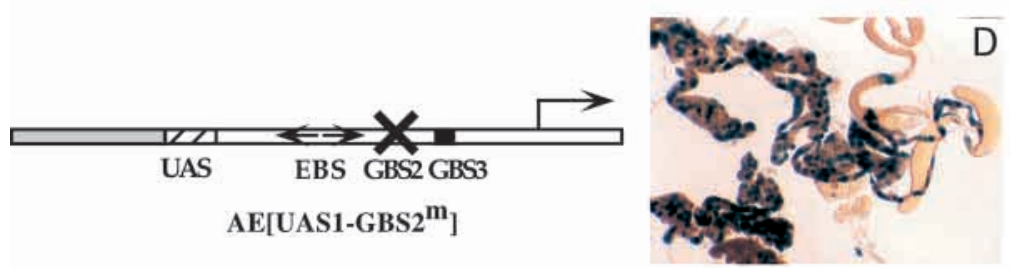

$\frac{\text { AE[UAS1-GBS2 }^{\text {m }} \text { ] }}{\text { GAL4 }^{\text {daG32 }}}$
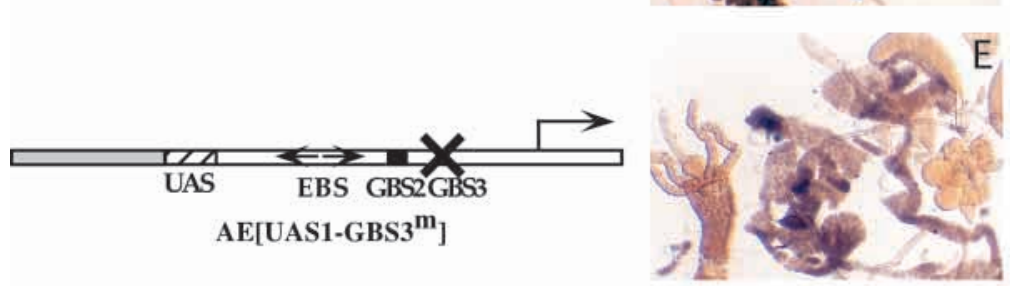

AE[UAS1-GBS3 ${ }^{\mathrm{m}}$ ]

GAL4 $^{\text {daG32 }}$
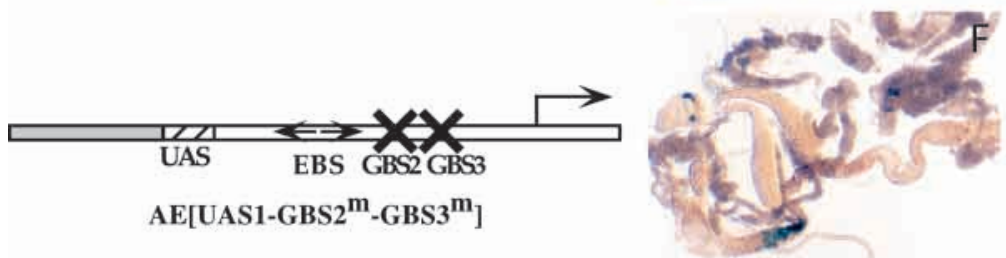

\section{$\underline{\text { AE[UAS1-GBS2 }}{ }^{\mathrm{m}}$-GBS3 $\left.^{\mathrm{m}}\right]$ \\ GAL4 $^{\text {daG32 }}$}

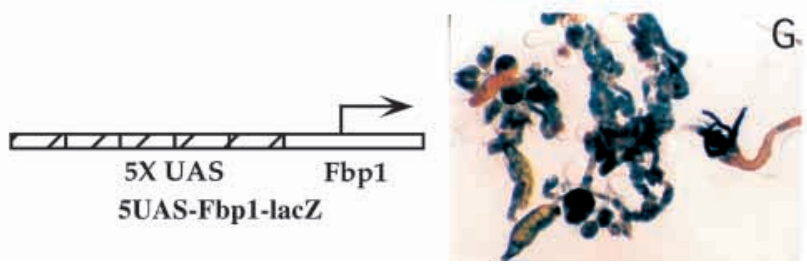

\section{UAS-Fbp1-lacZ \\ GAL4 ${ }^{\text {daG32 }}$}

Fig. 2. dGATAb is required for the specific transactivation of the Fbpl EcRU by GAL4. Late third instar larval tissues from transgenic lines with the indicated genotypes (right-hand side) were dissected and histochemically stained for $\beta$-galactosidase activity. Structures of the constructs in transgenic lines are depicted to the left-hand side, as in Fig. 1. Substituting UAS sites are indicated by hatched boxes. Disruptions of GBS2 and GBS3 are indicated by black crosses. Expression of the AE[UAS1] reporter construct in either the absence (A) or the presence

(B) of the GAL4daG32 driver is shown. 
Fig. 3. GAL4 activation of the Fbp1 EcRU is dependent upon the integrity of at least one of the two GBS1 or GBS3 sites. Late third instar larval tissues from transgenic lines with the indicated genotypes (right-hand side) were histochemically stained for $\beta$-galactosidase activity. Structures of the constructs in transgenic lines (left-hand side) are depicted as in Fig. 2. Expression of the AE[UAS2-3] reporter construct in either the absence (A) or the presence (B) of the GAL4 ${ }^{\mathrm{daG} 32}$ driver is shown.

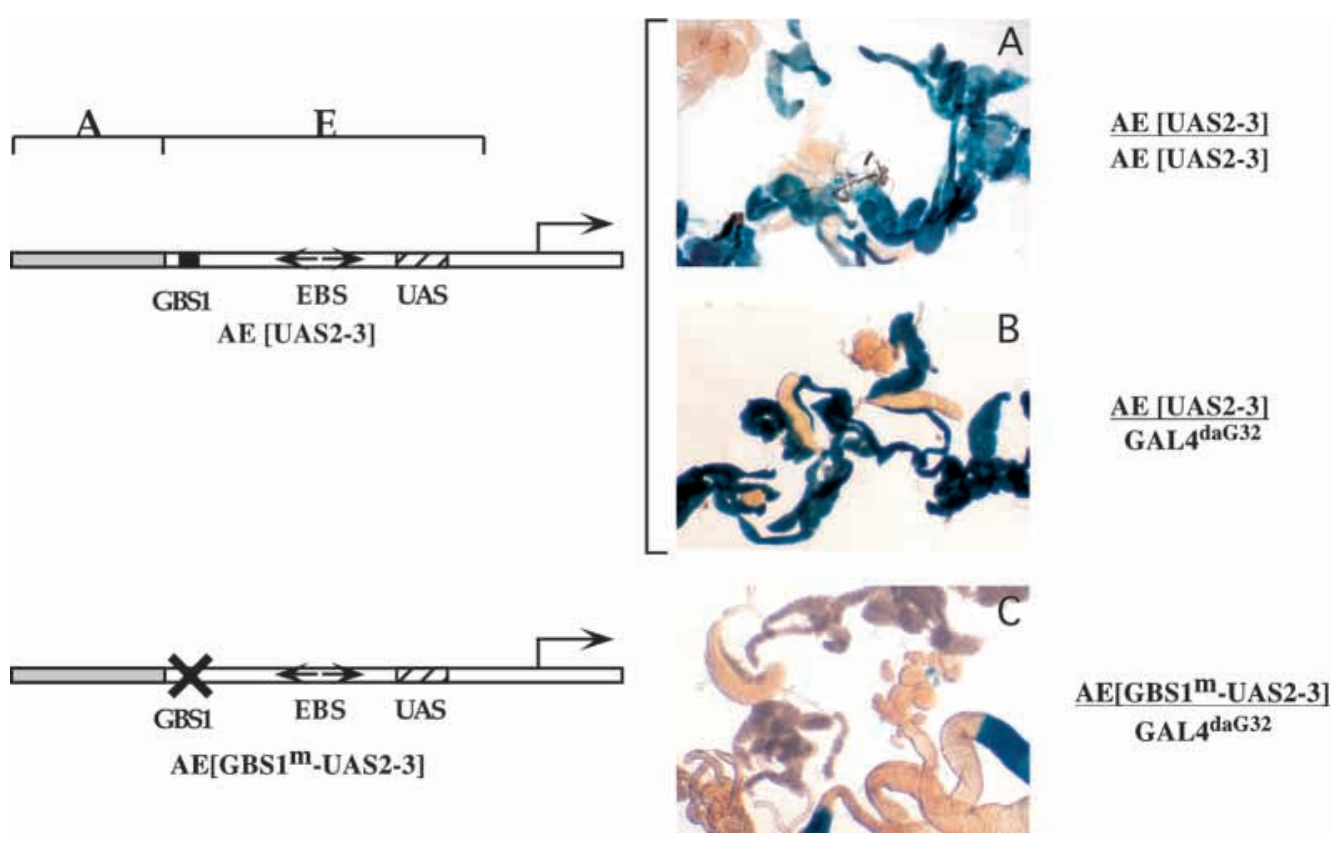

\section{GAL4 activation of the Fbp1 EcRU is silenced by the upstream element $A$}

The absence of expression of the AE[UAS1-UAS2-3] construct in a GAL4daG32 context led us to hypothesize that GAL4 activation was prevented by the silencing action exerted on the EcRU by a repressor whose activity would be normally antagonized by the binding of GATAb to either GBS1 or GBS3.

The possibility that target sites for this putative repressor were located between -68 and +80 in the minimal Fbpl promoter was ruled out, because of the full responsiveness of the 5UAS-Fbpl-lacZ construct to GAL4 in all but a few tissues throughout development (see Fig. 2G). Because it had been reported that the ecdysone receptor has a repressing activity in the absence of its ligand (Cherbas et al., 1991; Dobens et al., 1991; Tsai et al., 1999) we tested whether the ecdysone receptor itself was the putative repressor by mutating the EcR/USP binding site (EBS) in the AE-UAS1-UAS2-3 construct. In a GAL4 $4^{d a G 32}$ context, the resulting AE[UAS1-

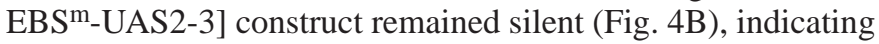
that the hormonal receptor was not involved in the silencing of GAL4 transactivation.

Finally, we examined the possibility that element A was the target for the putative repressor by deleting it in the AE[UAS1UAS2-3] construct. When placed in a GAL4daG32 genetic context, the resulting E[UAS1-UAS2-3] construct was expressed in all tissues but the central nervous system and gut, from embryogenesis to puparium formation (Fig. 4C; data not shown), indicating that element A probably contains a target sequence for a ubiquitous repressor strongly antagonizing transactivation by GAL4.

\section{The AEF-1 repressor binds to sequence $A$}

We noted that element A contains a putative binding site 5'CAACAA3' for the AEF-1 protein (Fig. 5), which had been identified as a factor that negatively regulates the Alcohol deshydrogenase $(A d h)$ and Yolk protein 1 and Yolk protein 2
(Yp1 and Yp2) genes of Drosophila melanogaster (An and Wensink, 1995; Falb and Maniatis, 1992a). AEF-1 is expressed throughout the Drosophila life cycle in all tissues examined, including the fat body (Falb and Maniatis, 1992b). This prompted us to examine the possibility that AEF-1 was the factor that mediated the repressing activity of element A.

The double-stranded oligonucleotide A1 that encompassed the putative AEF-1 site gave rise to the formation of a strong retarded complex $\mathrm{C} 1$ when used as a radioactive probe in a gel shift assay with a fat body nuclear extract (Fig. 5, lane 1). The formation of this complex was inhibited in the presence of a 200-fold molar excess of unlabeled oligonucleotide A1 indicating that it was sequence specific (Fig. 5, lane 2). In contrast, the formation of complex $\mathrm{C} 1$ was not inhibited by using, as a competitor, the oligonucleotide $\mathrm{A} 1^{\mathrm{m}}$, in which the putative AEF-1 binding site was mutated (Fig. 5, lane 3). When the binding reaction was performed in the presence of a rabbit polyclonal antibody raised against AEF-1, complex C1 was not formed and two slower migrating complexes S1 and S2 were revealed (Fig. 5, lane 4). Neither of these complexes was formed in the presence of the AEF-1 antibody alone (Fig. 5, lane 7). However, the migration of complex S1 but not that of complex S2 was further retarded in the presence of protein A, which has a strong affinity for IgGs (Fig. 5, lane 5) but does not bind to DNA by itself (Fig. 5, lane 6). This confirmed that $\mathrm{S} 1$ resulted from a specific supershift of complex $\mathrm{C} 1$ by the AEF-1 antibody and S2 from a nonspecific DNA binding activity that occurred when rabbit serum was incubated in the presence of fat body nuclear extract. Taken together, these results demonstrate that the AEF-1 repressor is present in fat body nuclear extracts and binds specifically to element A upstream of the Fbpl enhancer.

\section{AEF-1 silences the activation of the Fbp1 EcRU by GAL4}

In order to test whether AEF-1 was the repressor antagonizing in vivo the activation of the Fbpl EcRU by GAL4, we 


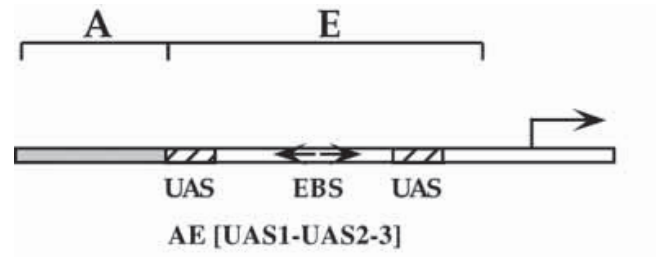

Fig. 4. AEF-1 silences the activation of the Fbpl EcRU by GAL4. Late third instar larval tissues from transgenic lines with the indicated genotypes (left-hand side) were histochemically stained for $\beta$ galactosidase activity. Structures of the constructs in transgenic lines (left-hand side) are depicted as in Fig. 2. Disruptions of the binding sites for EcR/USP and AEF-1 are indicated with black crosses.
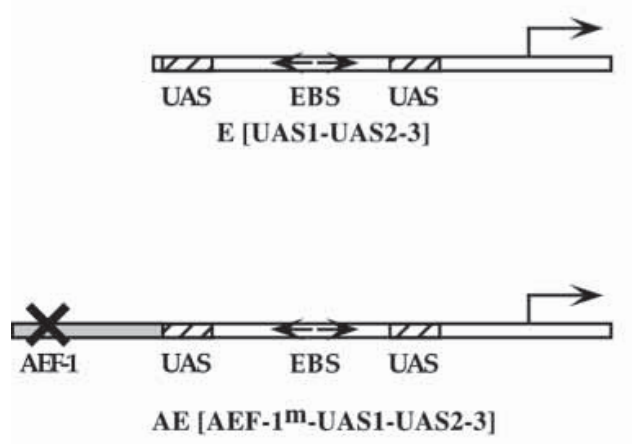

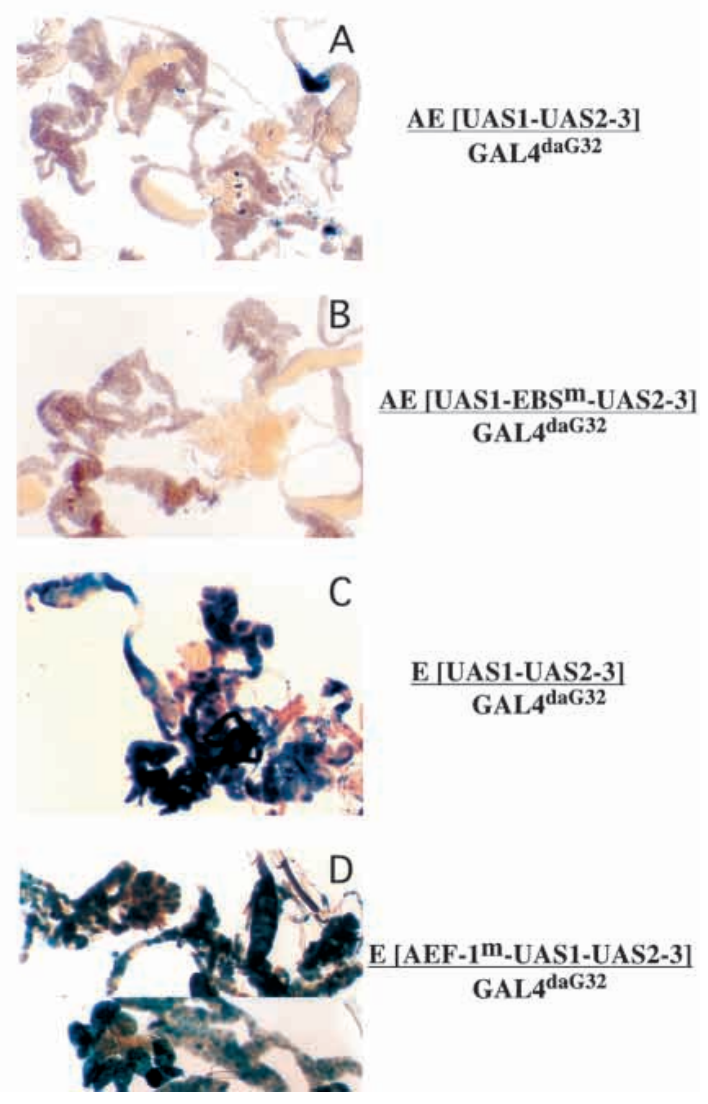

established transgenic lines for the AE[AEF-1 ${ }^{\mathrm{m}}$-UAS1-UAS23 ] construct bearing the mutation that abolished AEF-1 binding in gel shift experiments. In striking contrast to the AE[UAS1UAS2-3] construct (Fig. 4A), the AE[AEF-1 ${ }^{\text {m-UAS1-UAS2- }}$ $3]$ construct was expressed in the same way as the E[UAS1UAS2-3] construct in all tissues except the central nervous system and gut, and throughout development, when placed in a GAL4 $4^{d a G 32}$ genetic context (Fig. 4D; data not shown).

In summary, our experiments demonstrate that activation of the Fbpl EcRU by GAL4 is blocked by the binding of AEF-1 to element A. This silencing is relieved by the binding of GATAb to either GBS1 or GBS3.

\section{DISCUSSION}

\section{GATAb provides the competence necessary for Fbp1 EcRU to respond to GAL4}

Our results demonstrate that replacing putative binding sites of transactivating factors with a UAS site for the GAL4 yeast activator is a powerful alternative to the widely used disruption approach to the functional dissection of complex promoterdependent regulatory pathways. Our previous site inactivation experiments indicated that, of the three GATAb binding sites present in the Fbpl EcRU, only GBS1 was crucially required for the activity of the Fbpl EcRU in the third larval instar fat body in response to ecdysone, whereas GBS2 and GBS3 seemed to be dispensable (Brodu et al., 1999). Although this result indicated that binding of GATAb to the EcRU was essential, it gave no clue to the function of this factor other than that of transactivation. Because the replacement of GBS sites by a UAS site allowed a strong transcriptional activator to be targeted to the EcRU, it became feasible to examine whether GATAb was still required for the activity of the EcRU when GAL4 was present. The clear-cut patterns of expression of four constructs reported here provide an unambiguous answer to this question. The AE[GBS1 $\left.{ }^{\mathrm{m}}-\mathrm{UAS} 2-3\right]$ and AE[UAS1$\mathrm{GBS}^{\mathrm{m}}$ ] constructs, where the GBS1 and GBS3 sites, respectively, are inactivated, show a total lack of expression in the GAL4daG32 context, while the AE[UAS2-3] and AE[UAS1$\mathrm{GBS}^{\mathrm{m}}$ ] constructs, where one of these GBS sites remains intact, exhibit full expression in the same context. This provides a strong argument in support of the hypothesis that activation of the Fbpl EcRU by GAL4 crucially requires the binding of GATAb to at least one GBS site and indicates that this factor is specifically involved in a competence step that makes the EcRU responsive to transcriptional activators in the third larval instar fat body. Remarkably, the substitution approach reveals a functional redundancy of GBS1 and GBS3 in mediating this competence. In contrast, GBS2 did not appear to be able to support the same functional role. The ${ }^{5^{\prime}} \mathrm{GATT}^{3^{\prime}}$ core sequence of this site differs from the canonical ${ }^{5} \mathrm{GATA}^{3^{\prime}}$ core target sequence (Merika and Orkin, 1993) found in both GBS1 and GBS3 and we have shown that the GATAb binding affinity for GBS2 is lower than that for GBS1 and GBS3 (Brodu et al., 1999). Together, these data suggest that the apparent absence of any functional role for GBS2 in the Fbpl EcRU activity is related to its lower in vivo affinity for GATAb. 


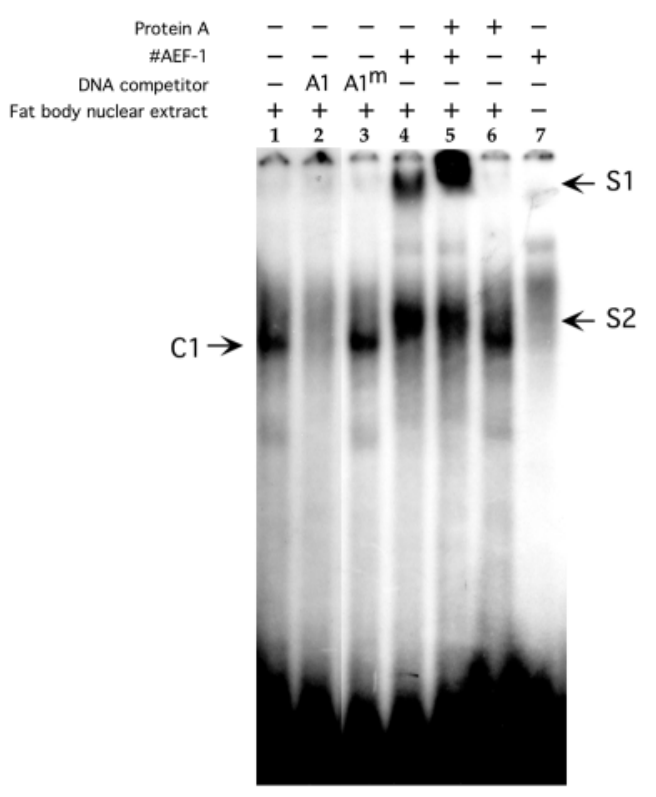

AEF-1

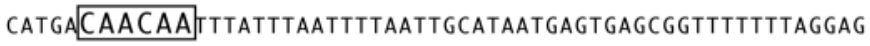
$\begin{array}{ll}\longrightarrow \text {-TCACCAC——1 } & \mathbf{A 1} \\ & \mathbf{A 1} \mathbf{m}^{\mathrm{m}}\end{array}$

Fig. 5. AEF-1 in third instar fat body nuclear extract binds to element A. Binding of proteins in a nuclear extract from late third instar fat body was analyzed in a gel shift assay using the element A (-194 to -138 ) as a probe in the presence or absence of competitor DNAs, AEF-1 antibody and protein A as indicated. C1, specific AEF-1 retarded complex; S1, AEF-1 supershifted complex; S2, nonspecific complex that formed when rabbit serum was incubated in the presence of fat body nuclear extract. The sequence of the element A probe and positions of the competitor DNAs are depicted at the bottom.

What are the mechanisms involved in the competence function of GATAb, as revealed by the UAS substitution approach? Numerous studies have shown that the yeast transcription factor GAL4 is able to activate reporter transgenic constructs under the control of UAS sites in all tissues, including those in which GATAb is not expressed (Brand and Perrimon, 1993; Phelps and Brand, 1998; Rorth, 1998). These data make it very unlikely that one of GATAb functions is to specifically potentiate the transactivating activity of GAL4 in the fat body tissue. The restriction of the expression of the AE[UAS1], AE[UAS2-3] and AE[UAS1-GBS2m] constructs to the fat body in a $G A L 4^{d a G 32}$ genetic context provides a strong argument in favor of the idea that specific sequences in these constructs target a potent ubiquitous repressor of GAL4 activity, which is antagonized solely in this tissue by means of a GATAb-dependent mechanism. The observation that the 5UAS-Fbpl-lacZ control construct is expressed throughout development in most tissues when crossed in the GAL4daG32 animals indicates that the Fbpl minimal promoter is fully responsive to GAL4 and does not contain any such repressor binding sequences. Similarly, the strong and fat-body specific expression of the AE[UAS1-UAS ${ }^{\mathrm{EBS}}$ ] construct excludes the
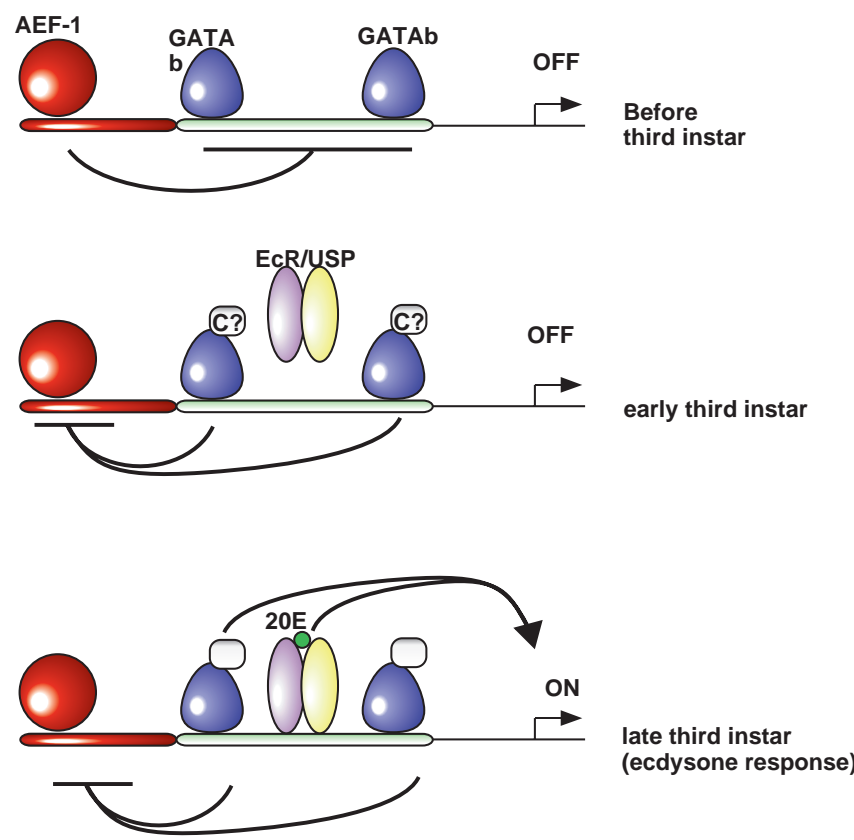

Fig. 6. Tentative model for the regulation of the Fbpl EcRU activity in the fat body during development. Before the third larval instar, AEF-1 bound to element A (red bar) silences the activity of the Fbpl enhancer (green bar). During the third larval instar, GATAb bound to GBS1 and GBS3 and associated with unidentified co-factors (C?) counteracts the repressing activity of AEF-1 in the fat body. Final activation of the Fbpl promoter during late third larval instar results from synergistic transactivation by the GATAb multiprotein complex at GBS1 and the EcR/USP ecdysone receptor activated by its 20-hydroxyecdysone (20E) ligand.

notion that the binding site for the EcR-USP receptor plays this role. In contrast our results clearly demonstrate that element A mediates the binding of the strong repressor AEF-1.

It is interesting to note that other instances of modulation of heterologous activators by Ultrabithorax-flanking regulatory sequences have been described (McCall and Bender, 1996; Szuts and Bienz, 2000). Together, these results provide strong support for the use of heterologous transactivators in transgenic systems as a tool to reveal and analyze the activity of cisregulatory modules in promoters.

\section{A novel function of the AEF-1 repressor}

The UAS substitution approach reveals that element A initially identified as a positively acting element (Lapie et al., 1993; Fig. 1) also possesses the properties of a negatively acting element. Deletion of element A from the completely inactive AE[UAS1UAS2-3] construct leads to the ubiquitous GAL4-driven expression of the resulting E[UAS1-UAS2-3] construct. The similar ubiquitous expression of the AE[AEF-1 ${ }^{\mathrm{m}}-\mathrm{UAS} 1$ UAS2-3] construct in which the AEF-1-binding site is disrupted, provides direct evidence that AEF-1 is the factor responsible for the complete blocking of GAL4 transactivation of the Fbpl EcRU. This is consistent with the initial characterization of AEF-1 as a repressor (Falb and Maniatis, 1992a) expressed throughout development in all tissues examined (Falb and Maniatis, 1992b). Our results, however, provide novel insight into the mechanisms whereby AEF-1 represses the transcription of its target genes. 
Two mechanisms of gene repression by transcriptional interference have been characterized so far for AEF-1. It has been shown that the binding of AEF-1 to the Adult Adh enhancer negatively regulates the $A d h$ gene by interfering with the binding of an activator of the C/EBP family to an overlapping site (Falb and Maniatis, 1992a; Falb and Maniatis, 1992b). A similar binding interference between AEF-1, C/EBP and the female-specific Doublesex protein was proposed for the downregulation of the yolk protein genes $Y p 1$ and $Y p 2$ (An and Wensink, 1995). More recently, Ren and Maniatis (Ren and Maniatis, 1998) showed that AEF-1 also binds to the initiator region (Inr) of the $A d h$ proximal promoter and represses transcription by a distinct mechanism thought to involve steric interference with the binding of general transcription factors. In contrast, our finding that AEF-1 is able to block the activation of the Fbpl EcRU by GAL4 targeted to a site more than $50 \mathrm{bp}$ downstream from the AEF-1 site, provides evidence that AEF-1 has yet another function, which is to repress enhancers at a distance.

Transcriptional repressors have been characterized by their range of action on promoters and enhancers (Gray and Levine, 1996b; Mannervik et al., 1999). Short-range repressors, including Snail, Knirp and Krüppel, interact over distances of 50-150 bp to inhibit, or quench, either transcriptional activators or the basal transcription complex (Gray and Levine, 1996a; Nibu et al., 1998a; Nibu et al., 1998b). These repressors share a conserved PXDLSXK sequence motif, responsible for interaction with the corepressor $\mathrm{dCtBP}$. In contrast, long-range repressors, including Dorsal and Hairy, act over distances of several kilobases to silence basal promoters (Barolo and Levine, 1997; Cai et al., 1996) and interact with the corepressor Groucho through a conserved WRPW motif. Our data suggest that AEF-1 belongs to the short-range repressor family. Whether AEF-1 is also able to act as a long-range repressor requires additional experiments.

The lack of PXDLSXK or WRPW motives in AEF-1 suggests that it mediates repression through an interaction with corepressors other than $\mathrm{dCtBP}$ and Groucho. Evidence that histone deacetylation plays a role in gene silencing has accumulated in recent years ( $\mathrm{Ng}$ and Bird, 2000). It has been shown in particular that the histone deacetylase Rpd3, and the Sin3A and SMRT/NcoR proteins are part of a corepressor complex of mammalian transcriptional repressors (reviewed in Ahringer, 2000). Similarly, Rpd3 and the Drosophila SMRT homolog SMRTER were shown to interact with Groucho (Flores-Saaib and Courey, 2000) and the unliganded EcR/USP ecdysone receptor (Tsai et al., 1999). In this context, a possible link between AEF-1 and complexes displaying a histone-deacetylase activity deserves investigation.

We were unable to demonstrate a direct protein-protein interaction between GATAb and AEF-1 in either an in vitro GST pull-down assay or two-hybrid screens in yeast using GATAb as a bait (data not shown). In addition, it should be noted that GATAb exerts its antagonizing effect on AEF-1 even when bound to GBS3, as in the AE[UAS1] construct where this site is located more than $100 \mathrm{bp}$ downstream of the AEF1-binding site with a GAL4 binding at an intervening site. It appears thus very unlikely that the interaction between GATAb and AEF-1 is direct.

\section{GATAb performs two distinct functions in the tissue- specific regulation of the Fbp1 EcRU}

A functional hierarchy between the GBS1 and GBS3 sites emerges from our results. GBS3 is dispensable but can redundantly supply the AEF-1 antagonizing GATAb effect when GBS1 is non-functional or absent. In contrast, GBS1 is essential for the activity of the natural Fbpl EcRU. This suggests that GBS1 not only supplies the AEF-1 antagonizing GATAb function, but is also involved in mediating another GATAb function essential to the specific activity of the Fbpl EcRU.

The E construct remains expressed in a tissue-restricted manner in the late third instar fat body (see Fig. 1), indicating that the Fbpl enhancer can act independently of element A as an autonomous time- and tissue-specific positive element. Disruption of GBS1 in the E construct leads to its inactivation (V. B., unpublished). Consistently, UAS substitution of the GATAb-binding sites in the E[UAS1-UAS2-3] construct also results in its total inactivation in the absence of GAL4. Together, these results point out to a GBS1-mediated specific activating function of GATAb on the Fbpl enhancer, in addition to its AEF-1 antagonizing function (Fig. 6). It is worth noting that both these functions are effective in the third instar fat-body only, leading to a highly tissue-specific regulatory output. The expression and developmental functions of GATAb are not, however, restricted merely to the larval fat body tissue. It also plays roles in the embryonic development of the gut (Reuter, 1994a), hematopoietic lineage (Lebestky et al., 2000; Rehorn et al., 1996) and gonads (Moore et al., 1998; Riechmann et al., 1998). Consistently, we have shown that GATAb is expressed in a number of third larval instar tissues, gonads, gut, lymph glands and pericardial cells in addition to the fat body. Hence, it is clear that the fat body-specific regulation of $\mathrm{Fbpl}$ by GATAb does not rely solely on the mere presence of this factor in the fat body. That additional factors are probably involved in fat body specification besides GATAb is further supported by the observation that ubiquitous overexpression of GATAb leads to an ectopic expression of the AE Fbpl construct limited to two additional tissues only, gut and salivary glands (Brodu et al., 1999). Together, these data suggest that in the fat body GATAb co-factors strictly target its dual Fbpl regulatory functions (Fig. 6). Our previous observation that GATAb interacts in complexes with as yet unidentified factors in the fat body (Brodu et al., 1999) strongly supports this hypothesis. Whether these factors have any effect on the fat body-specific functions of GATAb remains, however, to be determined.

\section{A model for the spatial and temporal regulation of the Fbp1 EcRU}

An additional benefit of the UAS substitution approach is that it provides information about the temporal inputs controlling the regulatory element under study. We had shown previously that the $\mathrm{AE}\left[\mathrm{UAS}{ }^{\mathrm{EBS}}\right]$ construct in which the ecdysone receptor binding site had been replaced by a UAS site, was expressed in the fat body throughout the third larval instar in a GAL4daG32 context. Because this temporal profile was clearly different from the late third instar-restricted expression of the $\mathrm{AE}$ construct, it pointed to EcR/USP as an essential element in the temporal regulation of the Fbpl EcRU (Brodu et al., 1999). In contrast, the temporal expression profile of the AE[UAS1] 
construct in which the EcR/USP binding site is intact, is identical to that of the $\mathrm{AE}$ and E constructs in the GAL4daG32 context (Fig. 2 and data not shown). This result fully supports the idea that the ecdysone receptor plays the role of a hormonally controlled transcription timer, whose function is both essential for and prevalent in the activation of the Fbpl EcRU (Fig. 6). We have recently shown that a switch in Broad gene products in the fat body is involved in the capacity of the Fbpl EcRU to respond to this hormonal activation (Mugat et al., 2000).

Nevertheless, our results point out to another level of temporal regulation for the Fbpl EcRU. On the one hand, the observation that GAL4-driven expression of the AE[UAS ${ }^{\mathrm{EBS}}$ ] and AE[UAS1-UAS ${ }^{\mathrm{EBS}}$ ] constructs takes place throughout the third larval instar but remains restricted to this stage indicates that a stage-specific competence for transactivation of the Fbpl EcRU is acquired at the third larval instar, independently from the hormonal control. On the other hand, the complete inactivity of the AE[UAS1-GBS3 $\left.{ }^{\mathrm{m}}\right]$, AE[UAS1-GBS2 ${ }^{\mathrm{m}}$ $\mathrm{GBS}^{\mathrm{m}}$ ] and AE[UAS1-UAS2-3] constructs strongly suggests that this stage-specific competence is dependent upon GATAb. In addition, the AE[AEF-1 ${ }^{\mathrm{m}}$-UAS1-UAS2-3] construct, which is not submitted to AEF-1 repression and therefore GATAbindependent, is ubiquitously expressed in embryos and throughout larval development in the GAL4daG32 context. Together, these data reveal the role of the GATAb/AEF-1 interplay in the acquisition of the capacity of the Fbpl EcRU to respond to transactivation during the third larval instar. However both GATAb and AEF-1 are expressed well before the third instar during embryogenesis. GATAb itself performs essential regulatory functions during this period of early development. Hence, it is clear that the stage-specific activation of the Fbpl EcRU does not rely solely on the combined functions of AEF-1 and GATAb. It is conceivable that GATAb co-factors such as those mentioned above are themselves temporally restricted to the third larval instar and required to potentiate these functions at the appropriate stage (Fig. 6).

We are grateful to T. Maniatis for the gift of the anti-AEF-1 antibody and we thank A. Kropfinger for critical reading of the manuscript. V. B. was a doctoral fellow of the Ministère de la Recherche et de l'Enseignement. This work was supported by grants to C. A. from the Association pour la Recherche Contre le Cancer $\left(\mathrm{n}^{\circ} 5202\right)$ and to J.-A. L. from the Association pour la Recherche Contre le Cancer ( $\left.{ }^{\circ} 6294\right)$, the Ligue Nationale Contre le Cancer and the Centre National de la Recherche Scientifique.

\section{REFERENCES}

Ahringer, J. (2000). NuRD and SIN3 histone deacetylase complexes in development. Trends Genet. 16, 351-356.

An, W. and Wensink, P. C. (1995). Integrating sex- and tissue-specific regulation within a single Drosophila enhancer. Genes Dev. 9, 256-266.

Andres, A. J., Fletcher, J. C., Karim, F. D. and Thummel, C. S. (1993). Molecular analysis of the initiation of insect metamorphosis: a comparative study of Drosophila ecdysteroid-regulated transcription. Dev. Biol. 160, 388404.

Andres, A. J. and Thummel, C. S. (1992). Hormones, Puffs and flies: the molecular control of metamorphosis by ecdysone. Trends Genet. 8, 132138.

Andres, A. J. and Thummel, C. S. (1994). Methods for quantitative analysis of transcription in larvae and prepupae. Methods Cell Biol. 44, 565-573.
Antoniewski, C., Laval, M., Dahan, A. and Lepesant, J. A. (1994). The ecdysone response enhancer of the Fbp1 gene of Drosophila melanogaster is a direct target for the EcR/USP nuclear receptor. Mol. Cell. Biol. 14, 44654474.

Antoniewski, C., Mugat, B., Delbac, F. and Lepesant, J. A. (1996). Direct repeats bind the EcR/USP receptor and mediate ecdysteroid responses in Drosophila melanogaster. Mol. Cell. Biol. 16, 2977-2986.

Arnone, M. I. and Davidson, E. H. (1997). The hardwiring of development: organization and function of genomic regulatory systems. Development 124, 1851-1864.

Ashburner, M. (1989). Drosophila: A Laboratory Handbook. Cold Spring Harbor, NY: Cold Spring Harbor Laboratory Press.

Barolo, S. and Levine, M. (1997). Hairy mediates dominant repression in the Drosophila embryo. EMBO J. 16, 2883-2891.

Brand, A. H. and Perrimon, N. (1993). Targeted gene expression as a means of altering cell fates and generating dominant phenotypes. Development 118 , 401-415.

Brodu, V., Mugat, B., Roignant, J. Y., Lepesant, J. A. and Antoniewski, C. (1999). Dual requirement for the EcR/USP nuclear receptor and the dGATAb factor in an ecdysone response in Drosophila melanogaster. Mol. Cell. Biol. 19, 5732-5742.

Burmester, T., Antoniewski, C. and Lepesant, J. A. (1999). Ecdysoneregulation of synthesis and processing of Fat Body Protein 1, the larval serum protein receptor of Drosophila melanogaster. Eur. J. Biochem. 262, 49-55.

Cai, H. N., Arnosti, D. N. and Levine, M. (1996). Long-range repression in the Drosophila embryo. Proc. Natl. Acad. Sci. USA 93, 9309-9314.

Cherbas, L., Lee, K. and Cherbas, P. (1991). Identification of ecdysone response elements by analysis of the Drosophila Eip28/29 gene. Genes Dev. 5, 120-131.

Dobens, L., Rudolph, K. and Berger, E. (1991). Ecdysterone regulatory elements function as both transcriptional activators and repressors. Mol. Cell. Biol. 11, 1846-1853.

Falb, D. and Maniatis, T. (1992a). A conserved regulatory unit implicated in tissue-specific gene expression in Drosophila and man. Genes Dev. 6, 454465.

Falb, D. and Maniatis, T. (1992b). Drosophila transcriptional repressor protein that binds specifically to negative control elements in fat body enhancers. Mol. Cell. Biol. 12, 4093-4103.

Flores-Saaib, R. D. and Courey, A. J. (2000). Analysis of groucho-histone interactions suggests mechanistic similarities between groucho- and Tup1mediated repression. Nucleic Acids Res. 28, 4189-4196.

Gray, S. and Levine, M. (1996a). Short-range transcriptional repressors mediate both quenching and direct repression within complex loci in Drosophila. Genes Dev. 10, 700-710.

Gray, S. and Levine, M. (1996b). Transcriptional repression in development. Curr. Opin. Cell Biol. 8, 358-364.

Kirchhamer, C. V., Yuh, C. H. and Davidson, E. H. (1996). Modular cisregulatory organization of developmentally expressed genes: two genes transcribed territorially in the sea urchin embryo, and additional examples. Proc. Natl. Acad. Sci. USA 93, 9322-9328.

Koelle, M. R., Talbot, W. S., Segraves, W. A., Bender, M. T., Cherbas, P. and Hogness, D. S. (1991). The drosophila EcR gene encodes an ecdysone receptor, a new member of the steroid receptor superfamily. Cell 67, 5977.

Lapie, P., Nasr, F., Lepesant, J. A. and Deutsch, J. (1993). Deletion scanning of the regulatory sequences of the Fbp1 gene of Drosophila melanogaster using P transposase-induced deficiencies. Genetics 135, 801-816.

Laval, M., Pourrain, F., Deutsch, J. and Lepesant, J. A. (1993). In vivo functional characterization of an ecdysone-response enhancer in the proximal upstream region of the Fbpl gene of D. melanogaster. Mech. Dev. 44, 123-138.

Lebestky, T., Chang, T., Hartenstein, V. and Banerjee, U. (2000). Specification of Drosophila hematopoietic lineage by conserved transcription factors. Science 288, 146-149.

Lepesant, J. A., Maschat, F., Kejzlarovà-Lepesant, J., Benes, H. and Yanicostas, C. (1986). Developmental and ecdysteroid regulation of gene expression in the larval fat body of Drosophila melanogaster. Arch. Insect Biochem. Physiol. S1, 133-141.

Lucas, P. C. and Granner, D. K. (1992). Hormone response domains in gene transcription. Annu. Rev. Biochem. 61, 1131-1173.

Mannervik, M., Nibu, Y., Zhang, H. and Levine, M. (1999). Transcriptional coregulators in development. Science 284, 606-609.

McCall, K. and Bender, W. (1996). Probes of chromatin accessibility in the 
Drosophila bithorax complex respond differently to Polycomb-mediated repression. EMBO J. 15, 569-580.

Merika, M. and Orkin, S. H. (1993). DNA-binding specificity of GATA family transcription factors. Mol. Cell. Biol. 13, 3999-4010.

Moore, L. A., Broihier, H. T., Van Doren, M. and Lehmann, R. (1998). Gonadal mesoderm and fat body initially follow a common developmental path in Drosophila. Development 125, 837-844.

Mugat, B., Brodu, V., Kejzlarova-Lepesant, J., Antoniewski, C., Bayer, C. A., Fristrom, J. W. and Lepesant, J. A. (2000). Dynamic expression of broad-complex isoforms mediates temporal control of an ecdysteroid target gene at the onset of drosophila metamorphosis. Dev. Biol. 227, 104117.

Ng, H. H. and Bird, A. (2000). Histone deacetylases: silencers for hire. Trends Biochem. Sci. 25, 121-126.

Nibu, Y., Zhang, H., Bajor, E., Barolo, S., Small, S. and Levine, M. (1998a). dCtBP mediates transcriptional repression by Knirps, Kruppel and Snail in the Drosophila embryo. EMBO J. 17, 7009-7020.

Nibu, Y., Zhang, H. and Levine, M. (1998b). Interaction of short-range repressors with Drosophila CtBP in the embryo. Science 280, 101-104.

Phelps, C. B. and Brand, A. H. (1998). Ectopic gene expression in Drosophila using GAL4 system. Methods 14, 367-379.

Rehorn, K. P., Thelen, H., Michelson, A. M. and Reuter, R. (1996). A molecular aspect of hematopoiesis and endoderm development common to vertebrates and Drosophila. Development 122, 4023-4031.

Ren, B. and Maniatis, T. (1998). Regulation of Drosophila Adh promoter switching by an initiator- targeted repression mechanism. EMBO J. 17, 1076-1086.

Reuter, R. (1994a). The gene serpent has homeotic properties and specifies endoderm versus ectoderm within the Drosophila gut. Development 120, 1123-1135.

Reuter, R. (1994b). The gene serpent has homeotic properties and specifies endoderm versus ectoderm within the Drosophila gut. Development 120, 1123-1135.

Riechmann, V., Rehorn, K. P., Reuter, R. and Leptin, M. (1998). The genetic control of the distinction between fat body and gonadal mesoderm in Drosophila. Development 125, 713-723.

Rorth, P. (1998). Gal4 in the Drosophila female germline. Mech. Dev. 78, 113 118.

Sam, S., Leise, W. and Hoshizaki, D. K. (1996). The serpent gene is necessary for progression through the early stages of fat-body development. Mech. Dev. 60, 197-205.

Szuts, D. and Bienz, M. (2000). LexA chimeras reveal the function of Drosophila Fos as a context- dependent transcriptional activator. Proc. Natl. Acad. Sci. USA 97, 5351-5356.

Thomas, H. E., Stunnenberg, H. G. and Stewart, A. F. (1993), Heterodimerization of the Drosophila ecdysone receptor with retinoid X receptor and ultraspiracle. Nature 362, 471-475.

Tsai, C. C., Kao, H. Y., Yao, T. P., McKeown, M. and Evans, R. M. (1999). SMRTER, a Drosophila nuclear receptor coregulator, reveals that EcRmediated repression is critical for development. Mol. Cell 4, 175-186.

Wodarz, A., Hinz, U., Engelbert, M. and Knust, E. (1995). Expression of crumbs confers apical character on plasma membrane domains of ectodermal epithelia of Drosophila. Cell 82, 67-76.

Yao, T. P., Forman, B. M., Jiang, Z., Cherbas, L., Chen, J.-D., McKeown, M., Cherbas, P. and Evans, R. M. (1993). Functional ecdysone receptor is the product of EcR and Ultraspiracle genes. Nature 336, 476-479. 